\title{
SPECTRAL DECOMPOSITION OF QUASI-MONTEL SPACES
}

\author{
BERTRAM WALSH ${ }^{1}$
}

In [8] the author showed that Montel spaces have the property that all regular Borel spectral measures with values in their continuous-linear-transformation algebras are necessarily purely atomic. The purpose of this note is to make the observation that by virtue of a theorem of Bartle, Dunford and Schwartz [1] and Grothendieck [3], this property is shared by a significantly larger class of locally convex spaces, namely the quasi-Montel spaces of K. Kera [5]. This class includes the classical Banach space $l^{1}$ and its subspaces and the "gestufte Räume" of Köthe and their subspaces [6]. The result given in this note also has consequences, which we shall mention briefly, in the study of the singular operators of Kantorovitz [4].

Let $E[\mathfrak{T}]$ be a locally convex topological vector space, which will be assumed to be boundedly complete; recall that a spectral measure triple in $\mathscr{L}(E)$ is a triple $(X, \Im, \mu)$ where $X$ is a set, $\subseteq$ is a $\sigma$-algebra of subsets of $X$, and $\mu: \subseteq \rightarrow \mathfrak{L}(E)$ is an $\mathscr{L}(E)$-valued set function which is countably additive in the weak operator topology and for which $\mu(X)=1$ (the identity transformation) and for any $\delta, \epsilon \in \Im, \mu(\delta \cap \epsilon)$ $=\mu(\delta) \cdot \mu(\epsilon) .(X, \mathfrak{S}, \mu)$ is said to be equicontinuous if the values of $\mu$ on $\subseteq$ are, and to be Baire or Borel if $X$ is a compact Hausdorff space and $\subseteq=\mathfrak{B}_{0}$ or $\mathfrak{B}$, its $\sigma$-algebras of Baire or Borel sets respectively. A Borel spectral measure triple $(X, \mathfrak{B}, \mu)$ is said to be regular if $\left\langle\mu(\cdot) x, x^{\prime}\right\rangle$ is a regular Borel measure for each $x \in E$ and $x^{\prime} \in E^{\prime}$ (cf. [8, Proposition 3.18]). A point atom of a Borel measure is a point $\xi \in X$ with $\mu(\{\xi\}) \neq 0$. For $x \in E$, the cyclic subspace and real cyclic subspace generated by $x$, denoted by $\mathfrak{M}(x)$ and $\mathfrak{M}_{R}(x)$ respectively, are the smallest closed subspace and closed real subspace of $E$ respectively which contain $\{\mu(\delta) x\}_{\delta \in \mathcal{G}}$. [8, Proposition 3.15] shows that both $\mathfrak{M}_{R}(x)$ and $\mathfrak{M}(x)$ are complete locally convex spaces when $E$ is boundedly complete in $\mathfrak{T}$, [8, Proposition 3.13 et seq.] that $\mathfrak{M}_{\mathbb{R}}(x)$ is a complete vector lattice when ordered by taking its positive cone to be the closed convex cone generated by $\{\mu(\delta) x\}_{\delta \in \subseteq}$, and also that $\mathfrak{M}(x)=\mathfrak{M}_{R}(x) \oplus i \mathfrak{M}_{R}(x)$.

It is easy to see that only small modifications of the proof of $[8$, Theorem 4.1] suffice to yield a proof of the slightly stronger-looking

Presented to the Society, January 25, 1966 under the title Atomicity of spectral measures on certain spaces; received by the editors March 2, 1966.

1 Partially supported by NSF Grant GP-4563. 
1. Proposition. Let $E[\mathfrak{T}]$ be a boundedly complete locally convex space, $\left(X, \mathfrak{B}_{0}, \mu\right)$ an equicontinuous Baire spectral measure triple in $\mathfrak{L}(E)$, and $(X, \mathfrak{B}, \tilde{\mu})$ its unique extension to a regular Borel spectral measure (see [8, Proposition 3.18]). Suppose that for each idempotent $e \neq 0$ in the strong closure of $\{\mu(\delta)\}_{\delta \in \mathfrak{B}_{0}}$ there exists a nonzero $x \in e E$ for which the order interval $[-x, x] \subseteq \mathfrak{M}_{R}(x)$ is compact. Then $(X, \mathfrak{B}, \tilde{\mu})$ possesses atoms, hence point atoms, and their supremum (in the sense of $[8$, Proposition 3.17]) is the identity element of $\mathfrak{L}(E)$.

Indeed, taking $e=1$ one sees that there does exist a nonzero $x \in E$ for which $[-x, x] \subseteq \mathfrak{M}_{R}(x)$ is compact, whence $\tilde{\mu}$ possesses some point atoms as in the proof of Theorem 4.1 of [8]. Letting $f$ be the supremum (in the sense of $\left[8\right.$, Proposition 3.17]) of the projections $\{\tilde{\mu}(\{\xi\})\}_{\xi \in X}$, we then see that unless $f=1$ the hypothesis of this proposition can be applied to the idempotent $e=1-f$, yielding a nonzero $y \in e E$ for which the order interval $[-y, y] \subseteq \mathfrak{M}_{R}(y) \subseteq e E$ is compact, and thus as in [8] giving a point atom $\xi$ for which $\tilde{\mu}(\{\xi\}) \cdot(1-f) \neq 0$, which is absurd.

For a given equicontinuous spectral measure triple $(X, \mathfrak{S}, \mu)$, let $M$ and $M_{R}$ denote respectively the algebras of bounded complex- and real-valued $\subseteq$-measurable functions on $X$.

2. LEMMA. Let $E[\mathfrak{T}]$ be a boundedly complete locally convex space and $(X, \Im, \mu)$ an equicontinuous spectral measure triple in $\mathscr{L}(E)$. Then for each $x \in E$ the interval $[-x, x] \subseteq \mathfrak{M}_{R}(x)$ is the closure of the set $\left\{\int f d \mu(x)\left|f \in M_{R},\right| f \mid \leqq 1\right\}$.

Proof. Since the interval is closed it will suffice to show that the latter set is dense in it. Let $q$ be any seminorm on $E$ compatible with $(X, \subseteq, \mu)$ in the sense of $[8$, Proposition $2.3 \mathrm{ff}$. $]$; then $\mu$ induces a spectral measure $\hat{\mu}_{q}$ on $\hat{E}_{q}=\left(E / q^{-1}[0]\right)^{\wedge}$ for which the natural quotient map $z \rightarrow z_{q}$ of $E \rightarrow \hat{E}_{q}$ preserves the algebraic and lattice operations on cyclic subspaces [8, Lemma 3.12]. In particular, this quotient map sends $[-x, x] \subseteq \mathfrak{M}_{R}(x)$ in to $\left[-x_{q}, x_{q}\right] \subseteq \mathfrak{M}_{R}\left(x_{q}\right) \subseteq \hat{E}_{q}$. Since $\hat{E}_{q}$ is a Banach space, for each $y \in[-x, x]$ there exists $f \in M_{R},-1 \leqq f$ $\leqq 1$, with $y_{q}=\int f d \hat{\mu}_{q}\left(x_{q}\right) \quad$ [8, Theorem 3.9]; in other words, $y_{q}$ $=\left(\int f d \mu(x)\right)_{q}$, or $q\left(y-\int f d \mu(x)\right)=0$. Since there are enough compatible seminorms to generate $\mathfrak{I}$, this shows that $\left\{\int f d \mu(x)\left|f \in M_{R},\right| f \mid \leqq 1\right\}$ is dense in $[-x, x]$.

REMARK. It follows that in the event there exists a continuous norm on $\mathfrak{M}_{R}(x)$, one has $\left\{\int f d \mu(x)\left|f \in M_{R},\right| f \mid \leqq 1\right\}=[-x, x]$.

Now suppose that one is given a boundedly complete space $E[\mathfrak{T}]$ which is a quasi-Montel space in the sense of [5], i.e., its weakly compact subsets are $\mathfrak{T}$-compact, and suppose $\left(X, \mathfrak{B}_{0}, \mu\right)$ is an equicon- 
tinuous Baire spectral measure triple in $\mathscr{L}(E)$; let $(X, \mathfrak{B}, \tilde{\mu})$ be its regular Borel extension. Then for each $x \in E$ the mapping $f \rightarrow \int f d \mu(x)$ is a continuous linear mapping from $\mathfrak{C}_{R}(X)$ to $\mathfrak{M}_{R}(x)$ with the property that for each closed $\delta \subseteq X$ the linear functional on $E^{\prime}$ defined by $x^{\prime} \rightarrow \lim _{f}\left\langle\int f d \mu(x), x^{\prime}\right\rangle$, where $f$ runs through the naturally downwarddirected set $\left\{f \mid 0 \leqq f \in \mathcal{C}_{R}(X), f \geqq \chi_{\delta}\right\}$, is $\sigma\left(E^{\prime}, E\right)$-continuous, namely is just $x^{\prime} \rightarrow\left\langle\tilde{\mu}(\delta) x, x^{\prime}\right\rangle$, because the measure $\tilde{\mu}$ is regular. Therefore by $[3 \text {, Theorem } 6]^{2}$ this mapping is weakly compact, i.e., the closure of $\left\{\int f d \mu(x)\left|f \in \mathcal{C}_{R}(X),\right| f \mid \leqq 1\right\}$ is weakly compact, and since $E$ is quasi-Montel $\mathfrak{T}$-compact, in $E$. But even the weak compactness of the mapping $f \rightarrow \int f d \mu(x)$ implies that it can be extended by weak continuity to a map from $\mathfrak{C}_{R}(X)^{\prime \prime} \rightarrow \mathfrak{M}_{R}(x)$ which takes the unit ball of the former to the closure of $\left\{\int f d \mu(x)\left|f \in \mathcal{C}_{R}(X),\right| f \mid \leqq 1\right\}$ in the latter. In particular, then, $\left\{\int f d \mu(x) \mid f \mathfrak{B}\right.$-measurable, $\left.|f| \leqq 1\right\}$ is contained in a compact set, and thus its closure, which by Lemma 2 above is $[-x, x]$, is $\mathfrak{T}$-compact. We have proved

3. Theorem. If $E[\mathfrak{T}]$ is a boundedly complete quasi-Montel space, then every equicontinuous Baire spectral measure in $\mathscr{L}(E)$ has purely atomic regular Borel extension, i.e., the regular Borel extension possesses point atoms and the supremum of the projections corresponding to those point atoms is the identity.

Indeed, we have shown that the hypotheses of Proposition 1 are satisfied.

Moreover, since any equicontinuous $\sigma$-complete Boolean algebra of idempotents in $\mathscr{L}(E)$ can be realized as the values of a Baire spectral measure on its Stone space, we have [8, Corollary 4.6] available on boundedly complete quasi-Montel spaces as well: the proof given in [8] uses only atomicity of $\tilde{\mu}$.

4. Corollary. If $E$ is a boundedly complete quasi-Montel space, then every equicontinuous $\sigma$-complete Boolean algebra in $\mathcal{L}(E)$ has a purely atomic completion equal to its strong closure in $\mathfrak{L}(E)$.

Examples of the spaces we have been considering are abundant: any perfect Köthe sequence space $\lambda$, equipped with its normal topology, is a complete quasi-Montel space [6,pp. 416 and 419]. Thus 3 and 4 above apply to any such $\lambda$ or to any of its closed subspaces.

${ }^{2}$ Or, alternatively, by representing the complete subspace $\mathfrak{M}_{R}(x)$ as a projective limit of Banach spaces, then observing that the induced maps $\mathfrak{C}_{R}(X) \rightarrow \mathfrak{M}_{R}(x)$ $\rightarrow \mathfrak{M}_{R}\left(x_{q}\right)$ are weakly compact by [1, Theorem 3.2$]$, whence the map $\mathfrak{C}_{R}(X) \rightarrow \mathfrak{M}_{R}(x)$ is also. 
Furthermore, since perfect sequence spaces are weakly sequentially complete [6, p. 415], any homomorphism of a $\mathcal{C}(X), X$ compact Hausdorff, into $\mathscr{L}(\lambda)$ which sends the unit sphere of $\mathfrak{e}(X)$ to an equicontinuous subset of $\mathfrak{L}(\lambda)$ can be given by an equicontinuous Baire spectral measure as in [7], and that measure may then be extended to a uniquely determined regular Borel measure as in $[8$, Proposition 3.18]. In particular, any "gestufter Raum" of Köthe [6, p. 422] is complete, metrizable, separable and quasi-Montel in its normal topology, so any weakly continuous homomorphism of a $\mathfrak{e}(X)$ in to its linear-transformation algebra sends the unit sphere to an equicontinuous set and can be given by an equicontinuous regular Borel spectral measure, which must be purely atomic; similarly, for these spaces the hypothesis of equicontinuity for $\sigma$-complete Boolean algebras is automatically satisfied [8, Proposition 1.2], while completeness and $\sigma$-completeness are equivalent by separability and metrizability: any $\sigma$-complete Boolean algebra on such a space is complete and purely atomic with only countably many atoms. The simplest example of such a "gestufter Raum" is, of course, the classical Banach space $l^{1}$.

Theorem 3 takes the following form for operators which are "scalar" in the sense of Dunford [2] ("spectral" in the sense of [7]); the proof is the same as that of [8, Corollary 4.8].

5. Corollary. Let $E[\mathfrak{T}]$ be boundedly complete and quasi-Montel, $u$ a scalar operator with domain $D_{u}$ and equicontinuous spectral measure $\nu$ (defined on the Borel sets of $\mathbf{C}$ ) for which $u=\int z d \nu$. Then

$$
u x=\int z d \nu(x)=\sum_{\lambda \in \pi(u)} \lambda \nu(\{\lambda\}) x
$$

for every $x \in D_{u}(\pi(u)$ denotes the point spectrum of $u)$.

More generally, it is not difficult to see that every operator with real spectrum on a quasi-Montel Banach space which is spectral of finite type $n$ in the sense of Dunford is a singular operator of class $C^{n}$ in the sense of Kantorovitz [4, Definition 3.9]. Indeed, the representation

$$
T(f)=\sum_{j=0}^{n} \int f^{(j)}(s) d\left[\frac{N^{j}}{j !} E(s)\right]
$$

for the $C^{n}$-operational calculus of $T$ with spectral measure $E$ and nilpotent part $N$ shows that the measures

$$
\mu_{j}\left(\cdot \mid x, x^{\prime}\right)=(1 / j !)\left\langle N E(\cdot) x,\left(N^{\prime}\right)^{j-1} x^{\prime}\right\rangle
$$


for $j \geqq 1$ are purely atomic, thus a fortiori singular. A converse proposition, i.e., that singular operators on these spaces are spectral of finite type, would follow in the case of $l^{1}$ from a strengthened version of [4, Lemma 3.10] with the hypothesis of reflexivity replaced by that of weak sequential completeness.

\section{REFERENCES}

1. R. Bartle, N. Dunford and J. Schwartz, Weak compactness and vector measures, Canad. J. Math. 7 (1955), 289-305.

2. N. Dunford, Spectral operators, Pacific J. Math. 4 (1954), 321-354.

3. A. Grothendieck, Sur les applications linéaires faiblement compactes d'espaces $d u$ type $C(K)$, Canad. J. Math. 5 (1953), 129-173.

4. S. Kantorovitz, Classification of operators by means of their operational calculus, Trans. Amer. Math. Soc. 115 (1965), 194-224.

5. K. Kera, On quasi-Montel spaces, Proc. Japan Acad. 40 (1964), 633-637.

6. G. Köthe, Topologische lineare Räume. I, Springer, Berlin, 1960.

7. H. Schaefer, Spectral measures in locally convex algebras, Acta Math. 107 (1962), $125-173$.

8. B. Walsh, Structure of spectral measures on locally convex spaces, Trans. Amer. Math. Soc. 120 (1965), 295-326.

University of California, Los Angeles 